\title{
Correlation between ZBRK1/ZNF350 gene polymorphism and breast cancer
}

\author{
Jun Wu, Alibiati Eni, Eliar Roussuri and Binlin Ma* (B)
}

\begin{abstract}
Background: This study is to explore the relationship between the ZBRK1/ZNF350 (Zinc finger and BRCA1-interacting protein with KRAB domain-1; also known as zinc-finger protein 350) gene polymorphism and early-onset breast cancer.

Methods: The ZBRK1/ZNF350 gene exon detection analysis was performed with the direct sequencing and Snapshot methods in 80 cases of breast cancer (aged $\leq 40$ years old) and 240 healthy subjects (aged $\leq 40$ years old).

Results: Totally 9 sequence variants were detected, including 5 missense mutations and 4 synonymous mutations, located at EXON3, EXON4 and EXON5, respectively. The rs4987241 and rs3764538 variants were published for the first time, while the remaining variants had been reported before. There were significant differences in the frequency distribution of family history between the breast cancer and control groups. Moreover, there were significant differences in the CT genotype frequency at the rs 138898320 locus between the breast cancer and healthy control groups. Compared with the carriers of CC wild genotype at rs 138898320 , the risk of breast cancer was reduced by $88.3 \%$ in the CT mutant genotype carriers, with significant difference. In the stratification with no family history, compared with the carriers of CC wild genotype at rs138898320, significant differences were observed for the CT mutant genotype carriers. In the stratification with family history, there was no significant difference in the variation of rs 138898320.
\end{abstract}

Conclusion: The rs 138898320 CT mutation genotype of ZBRK1/ZNF350 may reduce the risk of breast cancer, and the protecting effect would be increased in the stratification with no family history.

Trial registration Not applicable.

Keywords: Early-onset breast cancer, ZBRK1/ZNF350 gene, Single nucleotide polymorphism (SNP)

\section{Background}

Breast cancer is currently the most common malignancy in women worldwide [1]. Generally, breast cancer in young females is more aggressive, with poorer prognosis [2]. About $10 \%-15 \%$ of breast cancer cases are hereditary, which might be related to the mutations of $B R C A 1$ (Breast cancer susceptibility gene 1) and BRCA2 (Breast cancer susceptibility gene 2) [3]. However, the clinical significance of some variants is still not clear [4], and the

\footnotetext{
*Correspondence: mbldoctor@126.com
}

Surgical Department of Breast, Head and Neck Surgery, The Third Clinical

Medical College of Xinjiang Medical University (The Affiliated Tumor

Hospital), No. 789, Suzhou East Street, Urumqi 830011, Xinjiang, China disease may even be partly possible due to the genetic inheritance of multiple susceptible alleles with low penetrance [5].

It is shown that mutations in the $B R C A 1$ gene can easily cause breast cancer [6]. The $B R C A$ gene plays an important role in the homologous recombination mechanism of DNA repairing, and the germline $B R C A 1 / 2$ mutations will significantly increase the risk of breast cancer in females [7]. The most common types of disease-causing mutations in $B R C A 1 / 2$ are the nonsense mutations and frame-shift mutations. Gene mutations are inherited to offspring in an autosomal dominant manner [8]. The $B R C A 1$ has a wide range of functions. In addition to 
participating in the DNA repairing, it also participates in the transcription regulation. However, as a transcription regulator, BRCA1 protein itself cannot directly bind to its downstream genes and a transcription factor with the DNA sequence-specific binding ability is needed to help BRCA1 protein to bind to the downstream genes, thus exerting regulatory functions $[9,10]$.

ZBRK1 (Zinc finger and BRCA1-interacting protein with KRAB domain-1), also known as zinc-finger protein 350 (ZNF350), is a transcriptional suppressor gene, which can specifically recognize a 15 -bp DNA sequence, namely GGGxxxCAGxxxTTT (x represents any base) $[11,12]$. This sequence exists in the third intron of the downstream gene DNA damage response gene GADD45A regulated by BRCA1. BRCA1 binds to GADD45A through ZBRK1/ZNF350 to jointly inhibit the downstream regulatory genes (such as GADD45A), achieving the transcriptional regulation by BRCA1 [12, 13]. The BRCA1/ZBRK1 complex may play a role in the aspartic acid metabolism [14], and the ZBRK1/ZNF350 gene mutations have been detected in both familial and sporadic breast cancer patients [5, 15]. Moreover, abnormal ZBRK1/ZNF350 gene expression levels have also been observed in human breast cancer tissues [16]. Therefore, the sequence variation of the ZBRK1/ZNF350 gene is likely to affect the interaction between its product and BRCA1, thus interfering with the function of the tumor suppressor. Accordingly, ZBRK1/ZNF350 has been considered to be a new breast cancer susceptibility gene [17]. In this study, the correlation between ZBRK1/ZNF350 exonic variant and breast cancer was investigated.

\section{Methods}

\section{Study subjects}

Totally 80 Han females with early-onset breast cancer (age of diagnosis $\leq 40$ years old), were included in this study, who were confirmed by the pathological diagnosis in the Affiliated Tumor Hospital of Xinjiang Medical University. The blood samples were collected from these patients. None of these patients received radiotherapy or chemotherapy before surgery, and other malignant tumors were excluded. On the other hand, the blood samples were collected from another 240 age-matched healthy Han female subjects in our hospital during the same period. Detailed clinical information, such as the general information and postoperative case report, was obtained. Family hereditary breast cancer must meet the following conditions: (1) Early-onset breast cancer (age of diagnosis $\leq 40$ years old); (2) having a family history of breast cancer (at least one of the primary and secondary family members suffering from breast cancer); (3) pathologically diagnosed as breast cancer; and (4) with an expert in breast cancer assigned to evaluate medical history. All enrolled patients signed the informed consent. This study was approved by the Ethics Committee of the Affiliated Tumor Hospital of Xinjiang Medical University.

\section{Sample storage and DNA extraction}

Totally $5 \mathrm{ml}$ peripheral blood was collected, followed by the EDTA anticoagulation, which was stored at $-80{ }^{\circ} \mathrm{C}$. DNA extraction was performed with the DNA extraction kits and enzymes, according to the manufacturers' instructions. The genomic DNA was extracted from the peripheral blood samples with the phenol/chloroform method, and the concentration and purity were determined with the Biophotometer. Totally $1 \mu$ l extracted DNA was subjected to the assessment with $1 \%$ agarose electrophoresis, and the concentration was diluted down to $5-10 \mathrm{ng} / \mu \mathrm{l}$.

\section{DNA sequencing for the case group}

The reference sequence used was from the gene database of NCBI (ZNF350 zinc finger protein 350 [Homo sapiens (human)]; Gene ID: 59,348; https://www.ncbi.nlm.nih. gov/gene/59348). Totally 11 primers were designed and amplified, covering the 2-kb $5^{\prime}$-promoter of the ZBRK1/ ZNF350 gene and gene coding sequence. The primers were designed with Primer3 online software, which were synthesized by the Shanghai Tianhao Biotechnology Co., Ltd., Shanghai, China, with the following sequences: P1, forward $5^{\prime}$-TAGGAAGAGGAGTGGCGTGAA- $3^{\prime}$ and reverse $5^{\prime}$-TGCTTCTAAACACAACATTTAAGAAA-3'; P2, forward 5'-AATTCAGGGTGACGAAGTGGT-3' and reverse 5'-GGAAGAGCAGAGCCAGAATTG-3'; P3, forward 5'-TGTGGGTCATCCTTTACTCTCCT-3' and reverse $5^{\prime}$-GAATGAATTGGAAATGGAAAAGGT -3'; P4, forward 5'-GTGGGTGTCTGCCTACCTGAT$3^{\prime}$ and reverse 5'-CTCCCAAACCTTCCACTGAGA-3'; P5, forward 5'-TGAGTGCCTAGCAGCAGTGAC-3' and reverse 5'-TGCATGGTGTCACAAAATTCAT-3'; P6, forward 5'-TGCACACAGCCTGTAGAGTGG-3' and reverse $5^{\prime}$-ATCTGTAACATCAAGGTTTTTCTC TT-3'; P7, forward 5'-AAGAGAAAAACCTTGATGTTA CAGAT- $3^{\prime}$ and reverse $5^{\prime}$-CTCACACTGGGAGACAGA TGAG-3'; P8, forward 5'-CACAACAAACTTTTACCC CAAATC- $3^{\prime}$ and reverse $5^{\prime}$-GAAAGGCTTTGCCAC ATTCAG-3'; P9, forward 5'-GGGGACTCCTTTCTT CATGCT- $3^{\prime}$ and reverse $5^{\prime}$-TTCCACATTCACTGC ACACAA-3'; P10, forward 5'-TGAATGTGGCAAAGC CTTTCT- $3^{\prime}$ and reverse 5'-CACATCTGACCACTG GCTGTC- $3^{\prime}$; and P11, forward 5'-CACACAAGGGAG AAACAAGAGG- $3^{\prime}$ and reverse $5^{\prime}$-CCACTTAAAAGT ACTTGGGCTTCC- $3^{\prime}$. Accordingly, a PCR reaction system was established. The obtained PCR products were subjected to the sequencing analysis with the Big Dye 
kit (version 3.1; ABI, Grand Island, NY, USA), which was loaded on the ABI3130. The sequencing file was analyzed with the Polyphred software.

\section{Snapshot typing for the control group}

Based on the sequencing results of the case group, the target sites were screened according to the tag site mutual substitution principle and the high frequency sites. The following sites were selected for detection: rs4986771, rs138898320, rs4987241, and rs2278415. For the multiplex PCR reaction, the primers were designed to amplify the fragments containing the above 4 sites, and 4 extension primers adjacent to the SNP sites were designed for the single base extension. Primers were designed using the online Primer3 software. The PCR products were obtained with the HotStarTaq multiplex PCR (203,203; Qiagen). Then the PCR products were purified by the shrimp alkaline enzyme (SAP) (M9910; Promega, Madison, WI, USA) and exonuclease I (EXO I) (X40520K; Epicentre), and the extension was performed with the SNaPshot Multiplex kit (ABI). The extension products were purified with the SAP, which were then loaded on the ABI3730xl. The data were obtained, and the SNP typing was analyzed with the GeneMapper 4.1 (Applied biosystems).

\section{Statistical analysis}

The SPSS 20.0 software was used for statistical analysis. The $x^{2}$ test was used to compare the counting data (expressed as $\mathrm{n}(\%)$ ) between the case and control groups, and the t-test was used to compare the measurement data (represented by mean $\pm S D$ ) between the case and control groups. The correlation between the $Z B E K 1$ gene polymorphism and the case or control group was tested by the $x^{2}$ test. The binary Logistic regression was used to analyze the ZBRK1/ZNF350 exon genotypes and breast cancer susceptibility, as well as the OR and confidence interval $(95 \% \mathrm{CI}) . \quad P<0.05$ was considered statistically significant.

\section{Results \\ ZBRK1/ZNF350 gene mutation detection}

The ZBRK1/ZNF350 gene mutation detection was performed for the case and control groups. For the case group, the mutations in the exon region of the ZBRK1/ ZNF350 gene were detected with the direct sequencing method, and totally 9 sequence variants were detected (Table 1), including 5 missense mutations and 4 synonymous mutations located in EXON3, EXON4 and EXON5. These 5 missense mutations were as follows: rs4987241 (c.111G > A, EXON3); rs2278420 (c.197 T > C, EXON4); rs2278415 (c.1503A > T, EXON5); rs4986771 (c.1414 T>C, EXON5); and rs138898320 (c.100C > T, EXON3). These 4 synonymous mutations were as follows: rs4986773 (c.105 T > C, EXON3); rs4988334 (c.708 T > C, EXON5); rs3764538 (c.1119C > A, EXON5); and rs4986772 (c.1155A $>$ C, EXON5). On the other hand, for the control group, according to the sequencing results for the case group, the sequence variants were screened based on the tag site mutual substitution principle and the high frequency sites. The following sites were selected for detection: rs4986771, rs138898320, rs4987241, and rs2278415. Among them, rs4986771, rs4987241, rs2278415 had missense mutations, while rs138898320 had synonymous mutation (Table 2).

\section{General information of case and control groups}

Analysis of the general information of the case and control groups showed that, there were no statistically significant differences in BMI, age of menarche, age of first delivery, or times of pregnancies between the case and control groups (all $P>0.05$ ). However, significant

Table 1 ZBRK1/ZNF350 gene exon mutations and function prediction (case group)

\begin{tabular}{|c|c|c|c|c|c|c|c|}
\hline SNP No & Nucleotide change & SNP site & Location & Amino acid change & $\begin{array}{l}\text { Mutation } \\
\text { type }\end{array}$ & SIFT protein change & $\begin{array}{l}\text { SIFT } \\
\text { Prediction } \\
\text { score }\end{array}$ \\
\hline 1 & c.105 T>C & rs4986773 & EXON3 & p.Asp35Asp & $S$ & No effect & - \\
\hline 2 & c. $111 G>A$ & rs4987241 & EXON3 & p.Met37lle & $M$ & Impairment & 0.00 \\
\hline 3 & c.197T>C & rs2278420 & EXON4 & p.Leu66Pro & $M$ & Neutral & 0.47 \\
\hline 4 & c.708 T>C & rs4988334 & EXON5 & p.Cys236Cys & S & No effect & - \\
\hline 5 & C. $1119 C>A$ & rs3764538 & EXON5 & p.Pro373Pro & $S$ & No effect & - \\
\hline 6 & c. $1503 \mathrm{~A}>\mathrm{T}$ & rs2278415 & EXON5 & p.Arg501Ser & $M$ & Impairment & 0.04 \\
\hline 7 & C.1155A > C & rs4986772 & EXON5 & p.Thr385Thr & $\mathrm{S}$ & No effect & - \\
\hline 8 & c. $1414 \mathrm{~T}>\mathrm{C}$ & rs4986771 & EXON5 & p.Ser472Pro & $M$ & Neutral & 0.10 \\
\hline 9 & c. $100 C>T$ & rs138898320 & EXON3 & p.Arg34Trp & $M$ & Neutral & 0.09 \\
\hline
\end{tabular}

The SIFT prediction score $<0.05$ indicated that it affected the protein function. S, synonymous mutation; and M, missense mutation 
Table 2 ZBRK1/ZNF350 gene exon site mutations (control group)

\begin{tabular}{llllll}
\hline SNP No & SNP site & Location & Nucleotide change & Amino acid change & $\begin{array}{c}\text { Mutation } \\
\text { type }\end{array}$ \\
\hline 1 & rs4986771 & EXON5 & c.1414T $>C$ & p.Ser472Pro & M \\
2 & rs138898320 & EXON3 & c.100C $>T$ & p.Arg34Trp & S \\
3 & rs4987241 & EXON13 & c. 111G > A & p.Met37lle & M \\
4 & rs2278415 & EXON5 & c.1503A $>$ T & p.Arg501Ser & M \\
\hline
\end{tabular}

Table 3 General analysis of the case and control groups

\begin{tabular}{llll}
\hline & Case group $(\mathbf{N}=\mathbf{8 0})$ & $\begin{array}{l}\text { Control } \\
\text { group } \\
(\mathbf{N}=\mathbf{2 4 0})\end{array}$ & $\boldsymbol{P}$ \\
\hline BMI & $23.02 \pm 3.92$ & $23.02 \pm 3.65$ & 0.963 \\
Menarche age, yr & $13.28 \pm 1.59$ & $12.98 \pm 1.28$ & 0.099 \\
$\begin{array}{l}\text { Age of first delivery, yr } \\
\text { Number of pregnancy }\end{array}$ & $27.25 \pm 3.77$ & $27.33 \pm 3.24$ & 0.864 \\
$0-1$ & $34(42.5)$ & $98(40.8)$ & \\
$\geq 2$ & $46(57.5)$ & $142(59.2)$ & 0.793 \\
Family history of cancer & & & \\
Yes & $18(22.5)$ & $24(10.0)$ & \\
No & $62(77.5)$ & $216(90.0)$ & 0.006
\end{tabular}

The $x^{2}$ test was used to compare counting data (expressed as $n(\%)$ ) between the case and control groups, and the t-test was used to compare measurement data (represented by mean \pm SD) between the case and control groups

difference was observed in the frequency distribution of breast cancer family history between the case and control groups $(P=0.006)$ (Table 3$)$.

\section{Distribution of ZBRK1/ZNF350 gene exon genotype in case and control groups}

Analysis of ZBRK1/ZNF350 gene exon genotype distribution showed that, the CT genotype frequency of rs138898320 locus was significantly different between the case and control groups $\left(\chi^{2}=11.33 ; P=0.001\right)$. There was no statistically significant differences in the rs4986771, rs4987241, or rs2278415 genotype frequency distribution between the case and control groups $(P>0.05)$ (Table 4$)$.

\section{Logistic regression analysis of ZBRK1/ZNF350 gene exon genotype and breast cancer susceptibility}

Our results showed that, compared with the GG wild genotype carrier at the rs 4987241 site, the risk of breast cancer was increased by $21.6 \%(\mathrm{OR}=1.216 ; 95 \% \mathrm{CI}$ 0.436-3.389) for the GA carriers at the rs4987241 site. However, there was no statistical significance $(P=0.708)$. Moreover, compared with AA wild genotype carriers at the rs2278415 site, the risk of breast cancer for AT genotype carriers was increased by $17.5 \%$ $(\mathrm{OR}=1.175 ; 95 \%$ CI $0.693-1.992)$, while the risk of
Table 4 ZBRK1/ZNF350 gene exon genotype distribution in the case and control groups [ $\mathrm{n}(\%)]$

\begin{tabular}{lllll}
\hline Polymorphism & Case group $(\mathbf{N}=\mathbf{8 0})$ & $\begin{array}{l}\text { Control } \\
\text { group } \\
\mathbf{( N = 2 4 0}\end{array}$ & $\mathbf{X}^{\mathbf{2}}$ & $\boldsymbol{P}$ \\
\hline rs4986771 & & & \\
TT & $76(95.00)$ & $227(94.58)$ & & \\
CT & $4(5.00)$ & $13(5.42)$ & 0.00 & 1.000 \\
rs4987241 & & & & \\
GG & $75(93.75)$ & $222(92.50)$ & & \\
GA & $5(6.25)$ & $18(7.50)$ & 0.14 & 0.708 \\
rs138898320 & & & & \\
CC & $72(90.00)$ & $237(98.75)$ & & \\
CT & $8(10.00)$ & $3(1.25)$ & 11.33 & 0.001 \\
rs2278415 & & & & \\
AA & $49(61.25)$ & $131(54.58)$ & & \\
AT & $28(35.00)$ & $93(38.75)$ & 0.633 & 0.426 \\
TT & $3(3.75)$ & $16(6.67)$ & 0.647 & 0.412 \\
AT+TT & $31(38.75)$ & $109(45.42)$ & 1.084 & 0.298 \\
\hline
\end{tabular}

Note: The $x^{2}$ test was used to compare counting data (expressed as $\mathrm{n}(\%)$ ) between the case and control groups, and the t-test was used to compare measurement data (represented by mean \pm SD) between the case and control groups

breast cancer was increased by $83 \%$ for the TT genotype carriers $(\mathrm{OR}=1.833$; 95\% CI 0.52-6.463). However, no statistical significance was observed $(P=0.549$ and $P=0.346$, respectively). Furthermore, the risk of breast cancer was reduced by $67.8 \%(\mathrm{OR}=0.322 ; 95 \%$ CI, 0.079-1.319) for the CT genotype carriers than that for the TT genotype carriers at $\operatorname{rs} 4986771(P=0.115)$ (Table 5). In addition, compared with the carriers of CC wild genotype at rs138898320, the risk of breast cancer was significantly reduced by $88.3 \%$ in the CT mutation genotype carriers $(\mathrm{OR}=0.117 ; 95 \%$ CI $0.030-0.455)$ $(P=0.002)$ (Table 6). Moreover, family history stratification was performed. In the stratification without family history, compared with the carriers of CC wild genotype at rs138898320 site, significant difference was observed in the carriers of CT mutation genotype $(\mathrm{OR}=0.107 ; 95 \%$ CI $0.020-0.564)(P=0.008)$. On the other hand, in the stratification with family history, there was no significant difference in the variation 
Table 5 Logistic regression analysis of ZBRK1/ZNF350 gene exon genotype and breast cancer susceptibility

\begin{tabular}{|c|c|c|c|c|c|c|}
\hline Polymorphism & $\beta$ & SE $(\beta)$ & Wals & $P$ & OR & $95 \% \mathrm{Cl}$ \\
\hline \multicolumn{7}{|l|}{ rs4986771 } \\
\hline $\mathrm{TT}$ & & & & & 1.000 & \\
\hline $\mathrm{CT}$ & -1.133 & 0.719 & 2.481 & 0.115 & 0.322 & $0.079-1.319$ \\
\hline \multicolumn{7}{|l|}{ rs4987241 } \\
\hline GG & & & & & 1.000 & \\
\hline GA & 0.196 & 0.523 & 0.140 & 0.708 & 1.216 & $0.436-3.389$ \\
\hline \multicolumn{7}{|l|}{ rs138898320 } \\
\hline CC & & & & & 1.000 & \\
\hline $\mathrm{CT}$ & -2.172 & 0.690 & 9.904 & 0.002 & 0.114 & $0.029-0.441$ \\
\hline \multicolumn{7}{|l|}{ rs2278415 } \\
\hline AA & & & & & 1.000 & \\
\hline AT & 0.161 & 0.269 & 0.358 & 0.549 & 1.175 & $0.693-1.992$ \\
\hline $\mathrm{TT}$ & 0.606 & 0.643 & 0.889 & 0.346 & 1.833 & $0.520-6.463$ \\
\hline$A T+T T$ & 0.274 & 0.264 & 1.081 & 0.299 & 1.315 & $0.785-2.205$ \\
\hline
\end{tabular}

Table 6 Logistic regression analysis of ZBRK1/ZNF350 gene exon genotype and breast cancer susceptibility after correction

\begin{tabular}{|c|c|c|c|c|c|c|}
\hline Polymorphism & $\beta$ & SE $(\beta)$ & Wals & $P$ & OR & $95 \% \mathrm{Cl}$ \\
\hline \multicolumn{7}{|l|}{ rs4986771 } \\
\hline $\mathrm{TT}$ & & & & & 1.000 & \\
\hline CT & -0.416 & 0.604 & 0.474 & 0.491 & 0.660 & $0.202-2.154$ \\
\hline \multicolumn{7}{|l|}{ rs4987241 } \\
\hline GG & & & & & 1.000 & \\
\hline GA & 0.239 & 0.599 & 1.590 & 0.690 & 1.270 & $0.393-4.105$ \\
\hline \multicolumn{7}{|l|}{ rs138898320 } \\
\hline CC & & & & & 1.000 & \\
\hline $\mathrm{CT}$ & 2.026 & 0.710 & 8.346 & 0.004 & 7.581 & $1.918-26.963$ \\
\hline \multicolumn{7}{|l|}{ rs2278415 } \\
\hline AA & & & & & 1.000 & \\
\hline AT & -0.095 & 0.281 & 0.116 & 0.734 & 0.909 & $0.524-1.576$ \\
\hline $\mathrm{TT}$ & -0.747 & 0.656 & 1.300 & 0.254 & 0.474 & $0.131-1.712$ \\
\hline$A T+T T$ & -0.181 & 0.270 & 0.451 & 0.502 & 0.834 & $0.491-1.416$ \\
\hline
\end{tabular}

Table 7 Logistic regression analysis of rs138898320 locus and breast cancer susceptibility after family history stratification

\begin{tabular}{|c|c|c|c|c|c|c|c|}
\hline Family history & Polymorphism & $\beta$ & SE $(\beta)$ & Wals & $P$ & OR & $95 \% \mathrm{Cl}$ \\
\hline \multirow[t]{3}{*}{ Yes } & rs138898320 & & & & & & \\
\hline & CC & & & & & 1.000 & \\
\hline & $\mathrm{CT}$ & 1.056 & 1.267 & 0.694 & 0.405 & 2.875 & $0.240-34.462$ \\
\hline \multirow[t]{3}{*}{ No } & rs138898320 & & & & & & \\
\hline & CC & & & & & 1.000 & \\
\hline & CT & 2.439 & 0.830 & 8.633 & 0.003 & 11.464 & $2.253-58.344$ \\
\hline
\end{tabular}


of $\operatorname{rs} 138898320 \quad(\mathrm{OR}=0.185 ; 95 \%$ CI $0.018-1.941)$ $(P=0.159)$ (Table 7).

\section{Discussion}

ZBRK1/ZNF350 is located on chromosome 19 GRCh37. p2; 52,490,079-52,467,593 [18], covering about $10 \mathrm{~kb}$ and containing 4 exons [13], which encodes a protein of $60-\mathrm{kDa}$ [15]. This protein contains the highly conserved $\mathrm{KRAB}$ domain at the $\mathrm{NH} 2$ end, 8 continuous $\mathrm{C} 2 \mathrm{H} 2$ zinc finger motifs at the $\mathrm{COOH}$ end, and the CTRD domain. ZBRK1/ZNF350 protein inhibits transcription through CTRD with independent DNA binding [19]. The N-terminal KRAB domain of ZBRK1/ZNF350 protein binds to the repressor protein to form a transcriptional repression domain [20]. ZBRK1/ZNF350 protein is related to the occurrence of various human tumors [21]. GarciaClosas et al. [22] have conducted a meta-analysis based on the population of the United States (3368 cases and 2880 controls) and Poland (1995 cases and 2296 controls). They have found that there may be a weak link between the ZBRK1/ZNF350 rs4986771 locus and the risk of breast cancer. In the American population, compared to the wild-type homozygous genotype, heterozygotes at the ZBRK1/ZNF350 rs4986771 locus would suffer from increased risk of breast cancer, and the same genotype does not have significantly increased risk in the Polish population. A meta-analysis has showed that the rs4986771 gene mutation may have a protective effect on breast cancer [23]. However, in this study, our results showed that there was no statistically significant difference in the rs4986771 genotype frequency distribution between the case and control groups. The main reason for the difference between our conclusions and the metaanalysis [23] may be the sample size and study design. Their study was a meta-analysis performed on the population from the US (3368 cases and 2880 controls) and Poland (1995 cases and 2296 controls) with large sample size. However, the sample size in our original article was small. Further studies are needed.

In this study, through the direct sequencing of the exon region of the ZBRK1/ZNF350 gene in 80 cases of earlyonset (30-40 years old) breast cancer who were admitted to our hospital, a total of 9 sequence variants were detected. Garcia et al. [11] have performed the ZBRK1/ $Z N F 350$ gene sequencing on patients with primary breast cancer and have found 9 polymorphic sites, among which 7 sites were consistent with the sites found herein, namely rs2278420, rs2278415, rs4986771, rs4986773, rs4988334, rs3764538, and rs4986772. The SIFT software was used to predict the protein function, and our results showed that the sequence variants rs4987241 and rs2278415 in the breast cancer patients were harmful mutations, and one of the patients was the carrier of c.111G > A in rs4987241 aged 34 years old, who suffered from bilateral breast cancers (axillary lymph node metastasis at both sides, $3 / 22$ right breast and 1/26 left breast; the left breast was triple negative breast cancer). Another breast cancer patient also suffered from bilateral breast cancer, who was aged 37 years old, and both breasts were triple-negative breast cancers. However, in this study, there were no significant differences in the genotype frequencies of rs 4987241 and rs2278415 distribution between the case and control groups. However, a meta-analysis has shown that the risk of breast cancer is increased for the rs 2278415 gene mutation [23]. This phenomenon may be related to the sample size, and this site worth further investigation with enlarged sample sizes.

Polymorphism refers to a DNA sequence polymorphism caused by the substitution, insertion or deletion of a single nucleotide at the genome level. It is a common type of human heritable variation, accounting for more than $90 \%$ of known polymorphisms. When the minimum mutation frequency is greater than $1 \%$, it would be called polymorphism. As shown in Table 4, the mutation rate of a single base of the rs 138898320 allele was $3 / 480$, and the allele frequency was less than $1 \%$. Therefore, this locus may be a rare mutation rather than a polymorphism. In this study, it is worth noting that, compared with the carriers of CC wild genotype at rs 138898320 , the risk of breast cancer was reduced by $88.3 \%$ in carriers of CT mutant genotype, with statistically significant differences. In the stratification without family history, compared with the carriers of CC wild genotype at rs138898320, the CT mutation genotype carriers had protective effects on breast cancer, with statistically significant differences. However, in the stratification with family history, there was no significant difference in the variation of rs138898320 of ZBRK1/ZNF350 gene polymorphism.

Menstruation and fertility are important components of physiological reproductive factors. It has been shown that the menarche and age of first birth are related to the incidence of breast cancer. Early age of menarche and late age of first birth are important risk factors for breast cancer. In this study, our results showed that there was no statistically significant difference in BMI, age of menarche, age of first birth, or times of pregnancies between the case and control groups, and there was significant differences between the case and control groups with the breast cancer family.

\section{Conclusion}

In conclusion, the nine sequence variants found by the direct sequencing of the ZBRK1/ZNF350 gene exon region in this study were mostly consistent with previous findings, still with however some differences. To further clarify the relationship between the ZBRK1/ZNF350 
gene SNPs and breast cancer susceptibility, based on our findings herein, further in-depth studies with enlarged sample sizes concerning different populations are still needed.

\author{
Abbreviations \\ BRCA1: Breast cancer susceptibility gene 1; BRCA2: Breast cancer suscepti- \\ bility gene 2; ZBRK1: Zinc finger and BRCA1-interacting protein with KRAB \\ domain-1; ZNF350: Zinc-finger protein 350
}

\section{Acknowledgements}

None.

\section{Authors' contributions}

BM conceived and designed the experiments; JW, AE and ER conducted the experiments; JW analysed the results and wrote the manuscript. BM revised the manuscript. All authors reviewed the manuscript.

\section{Funding}

This work was supported by a grant from Natural Science Foundation of Xinjiang Uygur Autonomous Region (2017D01C408). The funding body had no role in the design of the study, collection, analysis, and interpretation of data or in writing the manuscript.

\section{Availability of data and materials}

The reference sequence used was from the gene database of NCBI (ZNF350 zinc finger protein 350 [Homo sapiens (human)]; Gene ID: 59,348; https:// www.ncbi.n/m.nih.gov/gene/59348). The datasets generated and/or analysed during the current study are deposited in European Variation Archive (EVA) under analysis accession number "ERZ1691763" (https://www.ebi.ac.uk/ena/ browser/view/ERZ1691763).

\section{Ethics approval and consent to participate}

All enrolled patients signed the informed consent. This study was approved by the Ethics Committee of the Affiliated Tumor Hospital of Xinjiang Medica University. No administrative permissions and/or licenses were acquired by our team to access the clinical/personal patient data used in our research.

\section{Consent for publication}

Not applicable.

\section{Competing interests}

The authors declare that they have no competing interests.

Received: 27 October 2020 Accepted: 21 December 2020

Published online: 06 January 2021

\section{References}

1. Bray F, Ferlay J, Soerjomataram I, Siegel RL, Torre LA, Jemal A. Global cancer statistics 2018: GLOBOCAN estimates of incidence and mortality worldwide for 36 cancers in 185 countries. CA Cancer J Clin. 2018:68:394-424

2. Peterlongo P, Chang-Claude J, Moysich KB, Rudolph A, Schmutzler RK, Simard J, et al. Candidate genetic modifiers for breast and ovarian cancer risk in BRCA1 and BRCA2 mutation carriers. Cancer Epidemiol Biomarkers Prev. 2015;24:308-16.

3. Aloraifi F, McCartan D, McDevitt T, Green AJ, Bracken A, Geraghty J. Protein-truncating variants in moderate-risk breast cancer susceptibility genes: a meta-analysis of high-risk case-control screening studies. Cancer Genet. 2015;208:455-63.

4. Arason A, Agnarsson BA, Johannesdottir G, Johannsson OT, Hilmarsdottir B, Reynisdottir I et al. The BRCA1 c.4096+3A>G Variant Displays Classical Characteristics of Pathogenic BRCA1 Mutations in Hereditary Breast and
Ovarian Cancers, But Still Allows Homozygous Viability. Genes (Basel). 2019;10.

5. Sigurdson AJ, Hauptmann M, Chatterjee N, Alexander BH, Doody MM, Rutter JL, et al. Kin-cohort estimates for familial breast cancer risk in relation to variants in DNA base excision repair, BRCA1 interacting and growth factor genes. BMC Cancer. 2004;4:9.

6. Zhang $X$, Li R. BRCA1-dependent transcriptional regulation: implication in tissue-specific tumor suppression. Cancers (Basel). 2018;10:513.

7. Liu C, Ma Y, Zhao X. Application of next-generation sequencing technology to detect breast cancer susceptibility genes BRCA1/2 and TP53 and PTEN germline mutations. Chin J Lab Med. 2019;42:98-103.

8. Roy R, Chun J, Powell SN. BRCA1 and BRCA2: different roles in a common pathway of genome protection. Nat Rev Cancer. 2011;12:68-78.

9. Fan $\mathrm{W}$, Jin S, Tong $\mathrm{T}$, Zhao H, Fan F, Antinore MJ, et al. BRCA1 regulates GADD45 through its interactions with the OCT-1 and CAAT motifs. J Biol Chem. 2002:277:8061-7.

10. Ouchi T, Monteiro AN, August A, Aaronson SA, Hanafusa H. BRCA1 regulates p53-dependent gene expression. Proc Natl Acad Sci USA. 1998;95:2302-6.

11. Yun J, Lee WH. Degradation of transcription repressor ZBRK1 through the ubiquitin-proteasome pathway relieves repression of Gadd45a upon DNA damage. Mol Cell Biol. 2003;23:7305-14.

12. Tan W, Zheng $L$, Lee WH, Boyer TG. Functional dissection of transcription factor ZBRK1 reveals zinc fingers with dual roles in DNA-binding and BRCA1-dependent transcriptional repression. J Biol Chem. 2004;279:6576-87.

13. Zheng L, Pan H, Li S, Flesken-Nikitin A, Chen PL, Boyer TG, et al. Sequencespecific transcriptional corepressor function for BRCA1 through a novel zinc finger protein, ZBRK1. Mol Cell. 2000;6:757-68.

14. Hong R, Zhang W, Xia X, Zhang K, Wang Y, Wu M, et al. Preventing BRCA1/ ZBRK1 repressor complex binding to the GOT2 promoter results in accelerated aspartate biosynthesis and promotion of cell proliferation. Mol Oncol. 2019;13:959-77.

15. Rutter JL, Smith AM, Dávila MR, Sigurdson AJ, Giusti RM, Pineda MA, et al. Mutational analysis of the BRCA1-interacting genes ZNF350/ZBRK1 and BRIP1/BACH1 among BRCA1 and BRCA2-negative probands from breastovarian cancer families and among early-onset breast cancer cases and reference individuals. Hum Mutat. 2003;22:121-8.

16. Garcia V, Domínguez G, García JM, Silva J, Peña C, Silva JM, et al. Altered expression of the ZBRK1 gene in human breast carcinomas. J Pathol. 2004;202:224-32.

17. Kiyotani K, Katagiri T. A commentary on Analysis of ZNF350/ZBRK1 promoter variants and breast cancer susceptibility in non-BRCA1/2 French Canadian breast cancer families. J Hum Genet. 2013:58:58.

18. Plourde KV, Labrie Y, Desjardins S, Belleau P, Ouellette G, Durocher F. Analysis of ZNF350/ZBRK1 promoter variants and breast cancer susceptibility in non-BRCA1/2 French Canadian breast cancer families. J Hum Genet. 2013;58:59-66.

19. Tan W, Kim S, Boyer TG. Tetrameric oligomerization mediates transcriptional repression by the BRCA1-dependent Kruppel-associated box-zinc finger protein ZBRK1. J Biol Chem. 2004;279:55153-60.

20. Urrutia R. KRAB-containing zinc-finger repressor proteins. Genome Biol. 2003:4:231

21. Nishitsuji H, Abe M, Sawada R, Takaku H. ZBRK1 represses HIV-1 LTRmediated transcription. FEBS Lett. 2012;586:3562-8.

22. García-Closas M, Egan KM, Newcomb PA, Brinton LA, Titus-Ernstoff L, Chanock $S$, et al. Polymorphisms in DNA double-strand break repair genes and risk of breast cancer: two population-based studies in USA and Poland, and meta-analyses. Hum Genet. 2006;119:376-88.

23. Zeng YF, Sang J. Five zinc finger protein 350 single nucleotide polymorphisms and the risks of breast cancer: a meta-analysis. Oncotarget. 2017:8:107273-82.

\section{Publisher's Note}

Springer Nature remains neutral with regard to jurisdictional claims in published maps and institutional affiliations. 